\title{
A CLOSED FORM FOR THE STIRLING POLYNOMIALS IN TERMS OF THE STIRLING NUMBERS
}

\author{
FENG QI AND BAI-NI GUO
}

\begin{abstract}
In the paper, by virtue of the Faá di Bruno formula and two identities for the Bell polynomial of the second kind, the authors find a closed form for the Stirling polynomials in terms of the Stirling numbers of the first and second kinds.
\end{abstract}

\section{Notation AND MAIN RESUlt}

It is common knowledge [1, p. 48] that the Bernoulli numbers $B_{j}$ are defined by

$$
\frac{z}{e^{z}-1}=\sum_{j=0}^{\infty} B_{j} \frac{z^{j}}{j !}=1-\frac{z}{2}+\sum_{j=0}^{\infty} B_{2 j} \frac{z^{2 j}}{(2 j) !}, \quad|z|<2 \pi .
$$

For some new developments in recent years about this topic, please refer to [2, 3, 6, 7, 10] and closely-related references therein.

The Stirling numbers of the first and second kinds $s(n, k)$ and $S(n, k)$ are important in combinatorial analysis, theory of special functions, and number theory. They can be generated by the rising factorial

$$
(x)_{n}=\prod_{k=0}^{n-1}(x+k)=\sum_{k=0}^{n} s(n, k) x^{k}
$$

and the exponential function

$$
\frac{\left(e^{x}-1\right)^{k}}{k !}=\sum_{n=k}^{\infty} S(n, k) \frac{x^{n}}{n !},
$$

see [1, p. 213, Theorem A] and [1, p. 206, Theorem A], and can be computed by explicit formulas

$$
s(n, k)=(-1)^{n+k}(n-1) ! \sum_{\ell_{1}=1}^{n-1} \frac{1}{\ell_{1}} \sum_{\ell_{2}=1}^{\ell_{1}-1} \frac{1}{\ell_{2}} \cdots \sum_{\ell_{k-2}=1}^{\ell_{k-3}-1} \frac{1}{\ell_{k-2}} \sum_{\ell_{k-1}=1}^{\ell_{k-2}-1} \frac{1}{\ell_{k-1}}
$$

for $1 \leq k \leq n$ and

$$
S(n, k)=\frac{1}{k !} \sum_{\ell=1}^{k}(-1)^{k-\ell}\left(\begin{array}{l}
k \\
\ell
\end{array}\right) \ell^{n}
$$

see [9, Corollary 2.3] and [1, p. 206]. For recent investigations on the Stirling numbers $s(n, k)$ and $S(n, k)$, please refer to [6, 7, 8, 9] and plenty of references cited therein.

The Stirling polynomials $S_{k}(x)$ are a family of polynomials that generalize the Bernoulli numbers $B_{k}$ and the Stirling numbers of the second kind $S(n, k)$. The

2010 Mathematics Subject Classification. Primary 11B83; Secondary 11B68, 33B10.

Key words and phrases. closed form; Stirling polynomial; Stirling number; Bernoulli number; Faá di Bruno's formula; Bell polynomial.

This paper was typeset using $\mathcal{A M S}_{\mathcal{M}}$-LATEX. 
Stirling polynomials $S_{k}(x)$ for nonnegative integers $k$ are defined by the generating function

$$
\left(\frac{t}{1-e^{-t}}\right)^{x+1}=\sum_{k=0}^{\infty} S_{k}(x) \frac{t^{k}}{k !} .
$$

The first six Stirling polynomials $S_{k}(x)$ for $0 \leq k \leq 5$ are

$$
\begin{gathered}
1, \quad \frac{x+1}{2}, \frac{3 x^{2}+5 x+2}{12}, \quad \frac{x^{3}+2 x^{2}+x}{8}, \\
\frac{15 x^{4}+30 x^{3}+5 x^{2}-18 x-8}{240}, \quad \frac{3 x^{5}+5 x^{4}-5 x^{3}-13 x^{2}-6 x}{96} .
\end{gathered}
$$

For more information on $S_{k}(x)$, see the papers [11, 12] and closely-related references therein.

In mathematics, a closed form is a mathematical expression that can be evaluated in a finite number of operations. It may contain constants, variables, four arithmetic operations, and elementary functions, but usually no limit.

In this paper, we find a closed form for the Stirling polynomials $S_{k}(x)$ in terms of the Stirling numbers $s(n, k)$ and $S(n, k)$ of the first and second kinds.

Our main result can be stated as the following theorem.

Theorem 1. For $k \geq 0$, the Stirling polynomials $S_{k}(x)$ can be computed by the closed form

$$
S_{k}(x)=(-1)^{k} k ! \sum_{m=0}^{k}\left[\sum_{\ell=m}^{k} \frac{s(\ell+1, m+1)}{(k+\ell) !} \sum_{i=0}^{\ell}(-1)^{i}\left(\begin{array}{c}
k+\ell \\
\ell-i
\end{array}\right) S(k+i, i)\right] x^{m} .
$$

\section{Proof of Theorem 1}

In combinatorics, the Bell polynomials of the second kind, or say, partial Bell polynomials, denoted by $\mathrm{B}_{n, k}\left(x_{1}, x_{2}, \ldots, x_{n-k+1}\right)$ for $n \geq k \geq 0$, are defined by

$$
\mathrm{B}_{n, k}\left(x_{1}, x_{2}, \ldots, x_{n-k+1}\right)=\sum_{\substack{1 \leq i \leq n, \ell_{i} \in\{0\} \cup \mathbb{N} \\ \sum_{i=1}^{n} i \ell_{i}=n \\ \sum_{i=1}^{n} \ell_{i}=k}} \frac{n !}{\prod_{i=1}^{n-k+1} \ell_{i} !} \prod_{i=1}^{n-k+1}\left(\frac{x_{i}}{i !}\right)^{\ell_{i}}
$$

See [1, p. 134, Theorem A]. They satisfy

$$
\mathrm{B}_{n, k}\left(a b x_{1}, a b^{2} x_{2}, \ldots, a b^{n-k+1} x_{n-k+1}\right)=a^{k} b^{n} \mathrm{~B}_{n, k}\left(x_{1}, x_{2}, \ldots, x_{n-k+1}\right)
$$

for complex numbers $a$ and $b$, see [1, p. 135], and

$$
\mathrm{B}_{n, k}\left(\frac{1}{2}, \frac{1}{3}, \ldots, \frac{1}{n-k+2}\right)=\frac{n !}{(n+k) !} \sum_{i=0}^{k}(-1)^{k-i}\left(\begin{array}{c}
n+k \\
k-i
\end{array}\right) S(n+i, i),
$$

see [3, Theorem 1 and Remark 1], [4, Remark 2.1], [5, p. 30], [8, p. 315], [10, Lemma 2.3], and [13, Example 4.2].

The Faà di Bruno formula can be described in terms of the Bell polynomials of the second kind $\mathrm{B}_{n, k}\left(x_{1}, x_{2}, \ldots, x_{n-k+1}\right)$ by

$$
\frac{\mathrm{d}^{n}}{\mathrm{~d} t^{n}} f \circ h(t)=\sum_{k=0}^{n} f^{(k)}(h(t)) \mathrm{B}_{n, k}\left(h^{\prime}(t), h^{\prime \prime}(t), \ldots, h^{(n-k+1)}(t)\right) .
$$

See [1, p. 139, Theorem C].

Taking $f(u)=u^{-(x+1)}$ and $u=h(t)=\frac{1-e^{-t}}{t}$ in the formula (5) and using the limit

$$
\lim _{t \rightarrow 0} u=\lim _{t \rightarrow 0} h(t)=\lim _{t \rightarrow 0} \frac{1-e^{-t}}{t}=1
$$


yield

$$
\begin{gathered}
\frac{\mathrm{d}^{k}}{\mathrm{~d} t^{k}}\left[\left(\frac{t}{1-e^{-t}}\right)^{x+1}\right]=\sum_{\ell=0}^{k} \frac{\langle-(x+1)\rangle_{\ell}}{u^{(x+1)+\ell}} \mathrm{B}_{k, \ell}\left(h^{\prime}(t), h^{\prime \prime}(t), \ldots, h^{(k-\ell+1)}(t)\right) \\
\rightarrow \sum_{\ell=0}^{k}\langle-(x+1)\rangle_{\ell} \mathrm{B}_{k, \ell}\left(\lim _{t \rightarrow 0} h^{\prime}(0), \lim _{t \rightarrow 0} h^{\prime \prime}(0), \ldots, \lim _{t \rightarrow 0} h^{(k-\ell+1)}(0)\right)
\end{gathered}
$$

as $t \rightarrow 0$, where

$$
\langle x\rangle_{n}=\prod_{\ell=0}^{n-1}(x-\ell)= \begin{cases}x(x-1) \cdots(x-n+1), & n \geq 1 \\ 1, & n=0\end{cases}
$$

is the falling factorial of $x \in \mathbb{R}$ for $n \in\{0\} \cup \mathbb{N}$. It is not difficult to see that

$$
\begin{aligned}
& \langle-(x+1)\rangle_{\ell}=\prod_{m=0}^{\ell-1}[-(x+1)-m]=(-1)^{\ell} \prod_{m=0}^{\ell-1}(x+m+1) \\
& =(-1)^{\ell} \prod_{m=1}^{\ell}(x+m)=\frac{(-1)^{\ell}}{x} \prod_{m=0}^{\ell}(x+m)=\frac{(-1)^{\ell}}{x}(x)_{\ell+1},
\end{aligned}
$$

where $(x)_{n}$ is defined by (1). Hence, it follows that

$$
\langle-(x+1)\rangle_{\ell}=\frac{(-1)^{\ell}}{x} \sum_{m=0}^{\ell+1} s(\ell+1, m) x^{m}, \quad \ell \geq 0 .
$$

Since

$$
h^{(\ell)}(t)=\int_{1 / e}^{1} s^{t-1}(\ln s)^{\ell} \mathrm{d} s \rightarrow \frac{(-1)^{\ell}}{1+\ell}, \quad t \rightarrow 0, \quad \ell \geq 0,
$$

by virtue of (3) and (4), we have

$$
\begin{aligned}
& \mathrm{B}_{k, \ell}\left(\lim _{t \rightarrow 0} h^{\prime}(0), \lim _{t \rightarrow 0} h^{\prime \prime}(0), \ldots, \lim _{t \rightarrow 0} h^{(k-\ell+1)}(0)\right)=\mathrm{B}_{k, \ell}\left(-\frac{1}{2}, \frac{1}{3}, \ldots, \frac{(-1)^{k-\ell+1}}{k-\ell+2}\right) \\
& =(-1)^{k} \mathrm{~B}_{k, \ell}\left(\frac{1}{2}, \frac{1}{3}, \ldots, \frac{1}{k-\ell+2}\right)=\frac{(-1)^{k+\ell} k !}{(k+\ell) !} \sum_{i=0}^{\ell}(-1)^{i}\left(\begin{array}{c}
k+\ell \\
\ell-i
\end{array}\right) S(k+i, i) .
\end{aligned}
$$

Accordingly, we obtain

$$
\begin{gathered}
\lim _{t \rightarrow 0} \frac{\mathrm{d}^{k}}{\mathrm{~d} t^{k}}\left[\left(\frac{t}{1-e^{-t}}\right)^{x+1}\right] \\
=\sum_{\ell=0}^{k}\left[\frac{(-1)^{\ell}}{x} \sum_{m=0}^{\ell+1} s(\ell+1, m) x^{m}\right]\left[\frac{(-1)^{k+\ell} k !}{(k+\ell) !} \sum_{i=0}^{\ell}(-1)^{i}\left(\begin{array}{c}
k+\ell \\
\ell-i
\end{array}\right) S(k+i, i)\right] \\
=\frac{(-1)^{k} k !}{x} \sum_{\ell=0}^{k} \frac{1}{(k+\ell) !}\left[\sum_{i=0}^{\ell}(-1)^{i}\left(\begin{array}{c}
k+\ell \\
\ell-i
\end{array}\right) S(k+i, i)\right]\left[\sum_{m=0}^{\ell+1} s(\ell+1, m) x^{m}\right] \\
=\frac{(-1)^{k} k !}{x} \sum_{m=0}^{k+1}\left[\sum_{\ell=m-1}^{k} \frac{s(\ell+1, m)}{(k+\ell) !} \sum_{i=0}^{\ell}(-1)^{i}\left(\begin{array}{c}
k+\ell \\
\ell-i
\end{array}\right) S(k+i, i)\right] x^{m}
\end{gathered}
$$

The explicit formula 2 is thus proved. The proof of Theorem 1 is complete. 


\section{REFERENCES}

[1] L. Comtet, Advanced Combinatorics: The Art of Finite and Infinite Expansions, Revised and Enlarged Edition, D. Reidel Publishing Co., Dordrecht and Boston, 1974.

[2] B.-N. Guo, I. Mezö, and F. Qi, An explicit formula for the Bernoulli polynomials in terms of the r-Stirling numbers of the second kind, Rocky Mountain J. Math. 46 (2016), no. 6, 1919-1923; Available online at http://dx.doi.org/10.1216/RMJ-2016-46-6-1919

[3] B.-N. Guo and F. Qi, An explicit formula for Bernoulli numbers in terms of Stirling numbers of the second kind, J. Anal. Number Theory 3 (2015), no. 1, 27-30; Available online at http://dx.doi.org/10.12785/jant/030105

[4] B.-N. Guo, F. Qi, Explicit formulas for special values of the Bell polynomials of the second kind and the Euler numbers, ResearchGate Technical Report (2015), http://dx.doi.org/ 10.13140/2.1.3794.8808

[5] B.-N. Guo and F. Qi, On inequalities for the exponential and logarithmic functions and means, Malays. J. Math. Sci. 10 (2016), no. 1, 23-34.

[6] B.-N. Guo and F. Qi, Some identities and an explicit formula for Bernoulli and Stirling numbers, J. Comput. Appl. Math. 255 (2014), 568-579; Available online at http://dx.doi. org $/ 10.1016 / j$.cam.2013.06.020

[7] F. Qi, A new formula for the Bernoulli numbers of the second kind in terms of the Stirling numbers of the first kind, Publ. Inst. Math. (Beograd) (N.S.) 100 (114) (2016), 243-249; Available online at http://dx.doi.org/10.2298/PIM150501028Q

[8] F. Qi, Diagonal recurrence relations, inequalities, and monotonicity related to the Stirling numbers of the second kind, Math. Inequal. Appl. 19 (2016), no. 1, 313-323; Available online at http://dx.doi.org/10.7153/mia-19-23

[9] F. Qi, Explicit formulas for computing Bernoulli numbers of the second kind and Stirling numbers of the first kind, Filomat 28 (2014), no. 2, 319-327; Available online at http: //dx.doi.org/10.2298/FIL14023190

[10] F. Qi and R. J. Chapman, Two closed forms for the Bernoulli polynomials, J. Number Theory 159 (2016), 89-100; Available online at http://dx.doi.org/10.1016/j.jnt.2015.07.021

[11] A. Schreiber, Multivariate Stirling polynomials of the first and second kind, Discrete Math. 338 (2015), no. 12, 2462-2484; Available online at http://dx.doi.org/10.1016/j.disc. 2015.06 .008

[12] M. Ward, The representation of Stirling's numbers and Stirling's polynomials as sums of factorials, Amer. J. Math. 56 (1934), no. 1-4, 87-95; Available online at http://dx.doi. org/10.2307/2370916

[13] Z.-Z. Zhang and J.-Z. Yang, Notes on some identities related to the partial Bell polynomials, Tamsui Oxf. J. Inf. Math. Sci. 28 (2012), no. 1, 39-48.

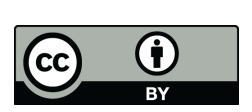

(C) 2017 by the authors; licensee Preprints, Basel, Switzerland. This article is an open access article distributed under the terms and conditions of the Creative Commons Attribution (CC BY) license http://creativecommons.org/licenses/by/4.0/).

(Qi) Institute of Mathematics, Henan Polytechnic University, Jiaozuo City, Henan Province 454010, China; College of Mathematics, Inner Mongolia University for Nationalities, Tongliao City, Inner Mongolia Autonomous Region 028043, China; Department of Mathematics, College of Science, Tianjin Polytechnic University, Tianjin City 300387, CHINA

E-mail address: qifeng618@gmail.com, qifeng618@hotmail.com

$U R L:$ https://qifeng618.wordpress.com

(Guo) School of Mathematics and Informatics, Henan Polytechnic University, JiaOzuo City, Henan Province 454010, China

E-mail address: bai.ni.guo@gmail.com, bai.ni.guo@hotmail.com

$U R L:$ http://www.researchgate.net/profile/Bai-Ni_Guo/ 